Developing Magnetorheological Finishing (MRF) Technology for the Manufacture of Large-Aperture Optics in Megajoule Class Laser Systems

J. A. Menapace

November 1, 2010

SPIE Laser Damage Conference

Boulder, CO, United States

September 26, 2010 through September 29, 2010 
This document was prepared as an account of work sponsored by an agency of the United States government. Neither the United States government nor Lawrence Livermore National Security, LLC, nor any of their employees makes any warranty, expressed or implied, or assumes any legal liability or responsibility for the accuracy, completeness, or usefulness of any information, apparatus, product, or process disclosed, or represents that its use would not infringe privately owned rights. Reference herein to any specific commercial product, process, or service by trade name, trademark, manufacturer, or otherwise does not necessarily constitute or imply its endorsement, recommendation, or favoring by the United States government or Lawrence Livermore National Security, LLC. The views and opinions of authors expressed herein do not necessarily state or reflect those of the United States government or Lawrence Livermore National Security, LLC, and shall not be used for advertising or product endorsement purposes. 


\title{
Developing Magnetorheological Finishing (MRF) Technology for the Manufacture of Large-Aperture Optics in Megajoule Class Laser Systems
}

\author{
Joseph A. Menapace* \\ Lawrence Livermore National Laboratory, \\ 7000 East Avenue, L-491, Livermore, CA 94550-9234 USA \\ *menapace1@1lnl.gov; phone (925) 423-0829; fax (925) 423-0792
}

This work performed under the auspices of the U.S. Department of Energy

by Lawrence Livermore National Laboratory under Contract DE-AC52-07NA27344

\begin{abstract}
Over the last eight years we have been developing advanced MRF tools and techniques to manufacture meter-scale optics for use in Megajoule class laser systems. These systems call for optics having unique characteristics that can complicate their fabrication using conventional polishing methods. First, exposure to the high-power nanosecond and sub-nanosecond pulsed laser environment in the infrared $\left(>27 \mathrm{~J} / \mathrm{cm}^{2}\right.$ at $\left.1053 \mathrm{~nm}\right)$, visible $\left(>18 \mathrm{~J} / \mathrm{cm}^{2}\right.$ at $\left.527 \mathrm{~nm}\right)$, and ultraviolet $\left(>10 \mathrm{~J} / \mathrm{cm}^{2}\right.$ at $351 \mathrm{~nm}$ ) demands ultra-precise control of optical figure and finish to avoid intensity modulation and scatter that can result in damage to the optics chain or system hardware. Second, the optics must be super-polished and virtually free of surface and subsurface flaws that can limit optic lifetime through laser-induced damage initiation and growth at the flaw sites, particularly at $351 \mathrm{~nm}$. Lastly, ultra-precise optics for beam conditioning are required to control laser beam quality. These optics contain customized surface topographical structures that cannot be made using traditional fabrication processes. In this review, we will present the development and implementation of large-aperture MRF tools and techniques specifically designed to meet the demanding optical performance challenges required in largeaperture high-power laser systems. In particular, we will discuss the advances made by using MRF technology to expose and remove surface and subsurface flaws in optics during final polishing to yield optics with improve laser damage resistance, the novel application of MRF deterministic polishing to imprint complex topographical information and wavefront correction patterns onto optical surfaces, and our efforts to advance the technology to manufacture largeaperture damage resistant optics.
\end{abstract}

Keywords: Optical finishing, computer numerical control, magnetorheological finishing, KDP, MRF, nonaqueous MR fluids, carbonyl iron, magnetorheological fluids, continuous phase plates, subsurface damage

\section{INTRODUCTION}

High-powered laser systems utilized for inertial confinement fusion research, such as the National Ignition Facility (NIF) at Lawrence Livermore National Laboratory (LLNL), Laser MegaJoule (CEA) near Bordeaux, France, and OMEGA at the Laboratory for Laser Energetics require large-aperture optics $(430$ x $430 \mathrm{~mm}$ and larger) whose performance specifications present unique manufacturing challenges that call for advanced finishing techniques and processes. One of the challenges for inertial confinement fusion (ICF) experiments in these systems is the requirement for different laser beam shapes at the target plane to get the desired pressure and temperature profiles needed to initiate fusion. In addition to the different laser beam shapes needed, precise characterization and control of the laser beam illumination at the target plane is essential to ensure uniformity in energy and momentum transfer to the target. Large-aperture continuous phase 
plates (CPPs) with ultra-precise surface topography were designed to meet this challenge; however, a manufacturing method was not available to fabricate the optics with high efficiency and laser damage resistance (IR, visible, and UV). These laser systems also require large-aperture optics such as lenses and windows that need to perform with enhanced laser damage resistance, particularly in the UV. In many cases, these optics are subjected to an environment where temporal UV laser pulses of about 3-nsec with average fluences of $8 \mathrm{~J} / \mathrm{cm}^{2}$ and peak fluences between 12 and $15 \mathrm{~J} / \mathrm{cm}^{2}$ are used. To operate effectively and reliably, the optics not only have to be manufactured with precise optical figure and finish, they also have to be free of bulk and surface artifacts to reduce the risk of laser-induced damage in this hostile environment. To meet these challenges, new optical fabrication tools, diagnostic tools, and manufacturing processes had to be designed and developed.

Since 2002, LLNL has focused on developing and deploying Magnetorheological Finishing (MRF) tools and techniques to meet NIF's needs for advanced optics. In this paper, we will present an overview of the development and implementation of large-aperture MRF tools and techniques specifically designed to meet the demanding optical performance challenges required in large-aperture high-power laser systems. In particular, we will address the advances made in MRF technology as they pertain to: 1) the manufacture of large-aperture CPPs using advanced MRF deterministic polishing techniques that imprint complex topographical information and wavefront correction patterns onto optical surfaces, 2) the exposure and removal of surface and subsurface flaws in optics during final polishing using MRF to yield optics with improved laser damage resistance, 3) the use of MRF as a diagnostic tool in the measurement and characterization of surface and near surface damage present in the various processing steps used to fabricate highperformance optics, and 4) the development of novel MRF tools and processes to polish extremely hard, extremely soft, single-crystal, polycrystalline, or water-soluble optical materials that serve as critical optical components in high-power laser systems. The details of the work performed in each of these areas can be found in the references provided. Those who are interested in pursuing additional information on the subjects are encouraged to refer to these papers.

\section{LARGE-APERTURE CPP IMPRINTING}

High-powered laser systems utilized for inertial confinement fusion research require precise characterization and control of the laser beam illumination at the target plane. A major portion of this work centers upon developing and engaging state-of-the-art technology to set the stage for creating nuclear fusion in a laboratory setting. Continuous phase plates (CPPs) form the vital and enabling portion of the optics chain used in these kilojoule- and megajoule-class laser systems because they make it possible to manipulate and control laser beam-shapes, energy distributions, and wavefront profiles $^{1,2,3,4,5,6,7}$. This prescribed beam characteristic control is made possible via manipulation of the incoming wavefront by the CPPs.

CPPs are large-aperture ultra-precision diffractive optics used in high-power kilojoule- and megajoule-class laser systems to adjust and fine tune a laser beam to a prescribed size and shape while maintaining the coherent properties of the laser light. CPPs function in combination with a focusing element to define the size and shape of the beam at focus, the wavefront characteristics (uniformity) at the focal plane, and the intensity of the beam within the focal spot.

As illustrated in Figure 1, a focusing element such as a lens results in laser light focused to a diffraction limited far-field spot of about $15 \mu \mathrm{m}$ in diameter at the focal plane (about a 30,000 times reduction in size). Additionally, it has a highly non-uniform intensity profile due to the limitations of the focusing element and light diffraction, which result in nonuniform illumination. Attempting to increase the spot size to about $100 \mu \mathrm{m}$ by defocusing the lens, which one would think could be a course of action, does make the far-field spot larger, but its shape and intensity profile remain ill-conditioned.

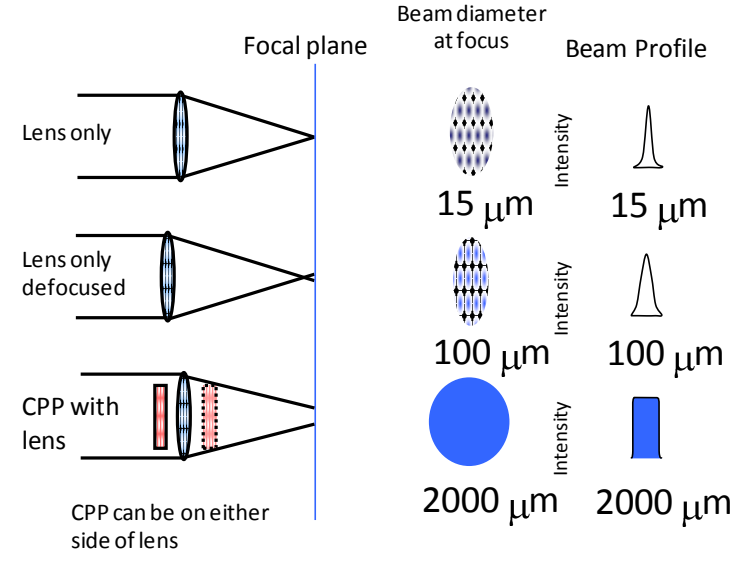

Figure 1: Focal plane characteristics with a lens focused to diffraction limit (top), with a lens moved from focus (middle), and with a lens and CPP combination set at focus (bottom). The CPP allows the focal plane to be sized and shaped while maintaining a intensity uniformity across the far-field spot. 
Introduction of a CPP into the optics chain that is specifically

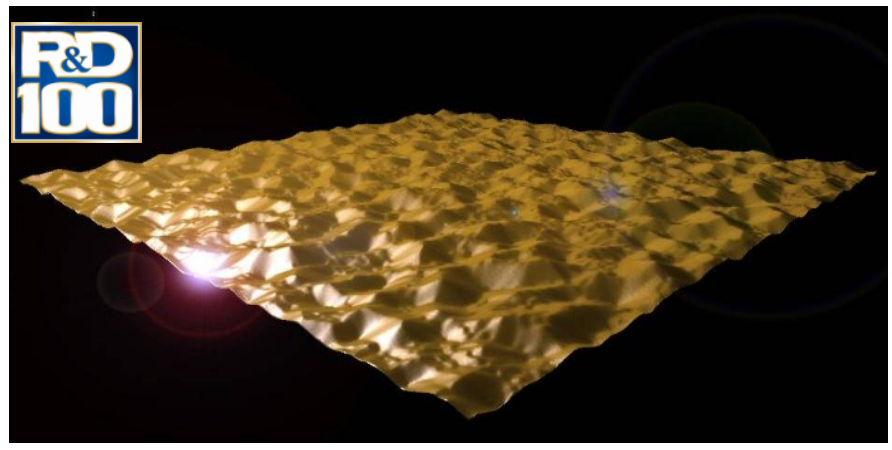

Figure 2: Continuously varying topographical CPP pattern with an $8.6 \mu \mathrm{m}$ peak-to-valley imprinted onto a $430 \times 430 \times 10 \mathrm{~mm}$ fused silica substrate using MRF. designed to yield the required far-field spot size and intensity uniformity in the focal plane solves the problem.

As CPPs fall into the category of diffractive optics, we take advantage of the apparent bending of light waves in response to small topographical changes on an optical surface. CPPs are made by imprinting a continuously varying phase profile onto an optical surface, as shown in Figure 2. These topographical changes are computer generated to achieve the required energy contours. This near-field topography is the key to enabling detailed control of the laser beam characteristics at the focal plane at high power. It can be designed to convert a square or circular laser beam footprint to an elliptical or circular spot of prescribed lateral dimensions. Other spot shapes, such as triangles, squares and closed polygons, are also possible. This continuously varying surface topography perturbs the incoming laser beam wavefront before, or after, passing through the final focusing element to yield a beam footprint at the focal plane with the desired characteristics.

MRF offers a direct approach for imprinting smooth topographical features onto optics without the use of lithographic masks or master plates. It is an advanced optical finishing process combining interferometry, precision equipment, and computer control. It utilizes a sub-aperture polishing tool, or removal function, generated by the interaction of a magnetic field and an iron-based MR fluid containing microscopic abrasive particles such as ceria or nano-diamonds. MRF is a deterministic polishing technique because the polishing tool effectively doesn't change. Because the removal function is interferometrically characterized and highly stable, the system can efficiently deliver high precision parts. Other advantages are that the polishing tool is easily adjusted, and conforms perfectly to the optical surface, enabling topographical polishing.

As imprinting of surface topography onto an optical surface such as that required for CPP's is a non-traditional application of this technology, we needed to understand the details of the relationship between removal functions and imprint fidelity, ${ }^{1}$ as illustrated in Figure 3. Development work in MRF CPP imprinting feasibility shows that the efficiency of the MRF process is directly tied to, and limited by, the removal function width. The removal function's width essentially limits the bandwidth of the surface topography due to Nyquist critical sampling issues. The removal function contains a band of Nyquist critical sampling frequencies between its narrowest and widest footprint dimensions. As spatial periods decrease, wider portions of the removal function become ineffective and lead to longer polishing times and greater material removal due to collateral polishing and topographical erasure.

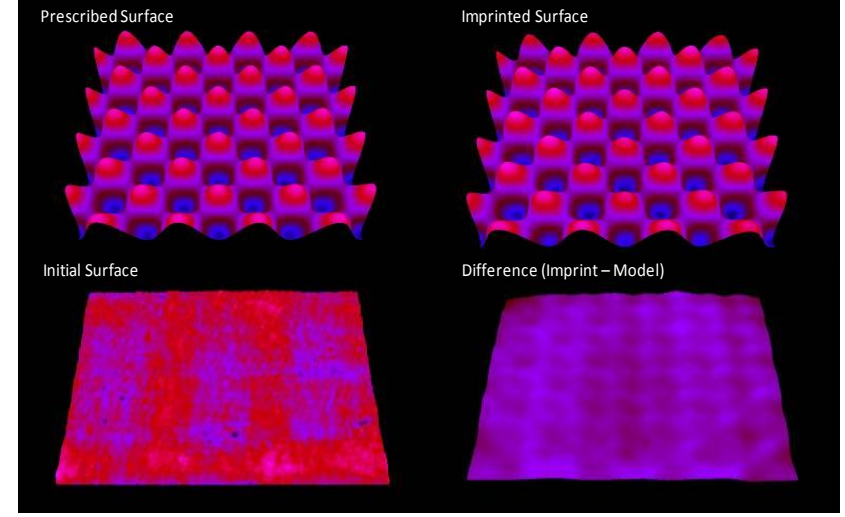

Figure 3: Example and comparison of an experimental sinusoidal profile with the modeled profile for MRF imprinting conducted using a $2.8-\mathrm{mm}$ removal function. (top-left) modeled surface, (top-right) Experimental surface topography, (bottomleft) the optical surface before MRF imprinting, and (bottomright) difference between the experimental and modeled surface. The rms of the difference between the experimental and the model topographies is $12 \mathrm{~nm}$ illustrating the fidelity of the process.

Several developments and observations were made during the diagnostic studies that lead to a process that could be used to imprint CPP's. First, imprinting should utilize a multi-pass MRF approach to obtain the desired topographical fidelity. Each pass should incorporate a different removal function size that maximizes material removal over the topographical frequencies being imprinted. Larger removal functions need to be used early in the fabrication process and small removal functions need to be used to perform final topographical correction. In-process interferometry should be an 
integral part of the process to optimize phasefront corrections. Superposition should be used during imprinting to provide for prescription simplification and process breakpoints necessary for testing.

MRF's deterministic polishing capability, wide array of available removal functions, and close interplay with interferometry enable imprinting of a diffractive phase structure that varies continuously across the whole beam aperture with no sharp discontinuities or phase anomalies. Material removal rates ranging between $0.025-1 \mathrm{~mm}^{3}$ per minute can produce an imprinted optic in 50-75 hours. The technology is capable of, and routinely produces, highly precise topographical profiles with errors of better than $30 \mathrm{~nm}$ rms over the optic aperture that yields highly efficiency plates (> 99 percent) whose characteristics are precisely defined. Optical surface finish is also maintained at better than 4.0 Angstroms rms roughness.

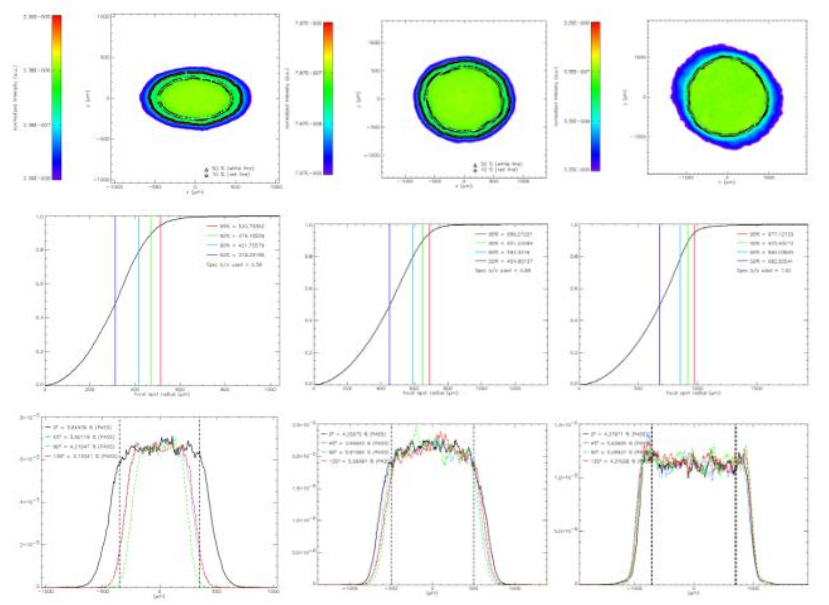

Figure 4: Examples of large-aperture CPPs manufactured and tested at LLNL. Left: 50-degree outer cone implosion CPP, ellipticity of 0.56 . Center: 23 -degree inner cone implosion CPP, ellipticity of 0.88 . Right: $2-\mathrm{mm}$ far-field spot illuminator CPP, ellipticity of 1.00 .

Figure 4 and Table 1 show the far-field characteristics for three different CPP types that have been designed and tested at LLNL. Twenty unique CPP designs have been manufactured and tested to date.

Imprinting large-aperture CPPs requires specialized MRF instruments capable of imprinting topographies on largeaperture optics. An integral part of our efforts for making CPPs a reality involves a large-aperture MRF tool, as illustrated in Figure 5. This machine, the world's largest and most precise MRF, is used to perform final finishing on CPP substrates and to imprint the necessary topography to manufacture a CPP. The large-aperture MRF system uses two wheels for efficiency and precision, one large $(370 \mathrm{~mm}$ diameter) and the other small (50 $\mathrm{mm}$ diameter), with optimized computer algorithms that together provide a greatly increased range of finishing options and range of topographical feature sizes that can be imprinted. The MRF process provides for a high level of versatility and speed in CPP manufacture as topographical polishing can be conducted by combining computer generated CPP and/or interferometric profiles with large MRF volumetric removal
Table 1: Examples of CPP performance parameters versus specification for large-aperture CPPs manufactured using MRF

\begin{tabular}{|l|c|c|}
\hline & Specification & Measured \\
\hline 50-degree outer cone implosion CPP & & \\
\hline 80\% Encircled Energy Radius $(\mu \mathrm{m})$ & $421.0 \pm 15.0$ & 421.8 \\
\hline 90\% Encircled Energy Radius $(\mu \mathrm{m})$ & $472.0 \pm 15.0$ & 476.2 \\
\hline 95\% Encircled Energy Radius $(\mu \mathrm{m})$ & $515.0 \pm 15.0$ & 520.8 \\
\hline Individual Lineout RMS $(\%)$ & 5.0 & 4.2 \\
\hline 2D RMS over Central Area $(\%)$ & 5.0 & 4.5 \\
\hline 50\% Ellipticity $(\mathrm{a}=451.2, \mathrm{~b}=252.4)$ & $0.56 \pm 0.1$ & 0.56 \\
\hline & & \\
\hline 23-degree inner cone implosion CPP & & \\
\hline 80\% Encircled Energy Radius $(\mu \mathrm{m})$ & $594.0 \pm 15.0$ & 592.3 \\
\hline 90\% Encircled Energy Radius $(\mu \mathrm{m})$ & $652.0 \pm 15.0$ & 651.2 \\
\hline 95\% Encircled Energy Radius $(\mu \mathrm{m})$ & $696.0 \pm 15.0$ & 696.2 \\
\hline Individual Lineout RMS $(\%)$ & 7.0 & 5.9 \\
\hline 2D RMS over Central Area $(\%)$ & 5.0 & 4.7 \\
\hline 50\% Ellipticity $(\mathrm{a}=451.2, \mathrm{~b}=252.4)$ & $0.88 \pm 0.1$ & 0.88 \\
\hline & & \\
\hline 2-mm far-field spot illuminator CPP & & \\
\hline 80\% Encircled Energy Radius $(\mu \mathrm{m})$ & $860.0 \pm 15.0$ & 860.0 \\
\hline 90\% Encircled Energy Radius $(\mu \mathrm{m})$ & $923.0 \pm 15.0$ & 925.4 \\
\hline 95\% Encircled Energy Radius $(\mu \mathrm{m})$ & $976.0 \pm 15.0$ & 977.1 \\
\hline Individual Lineout RMS $(\%)$ & 6.5 & 5.6 \\
\hline 2D RMS over Central Area $(\%)$ & 6.5 & 5.7 \\
\hline 50\% Ellipticity $(\mathrm{a}=451.2, \mathrm{~b}=252.4)$ & $1.02 \pm 0.1$ & 1.02 \\
\hline
\end{tabular}

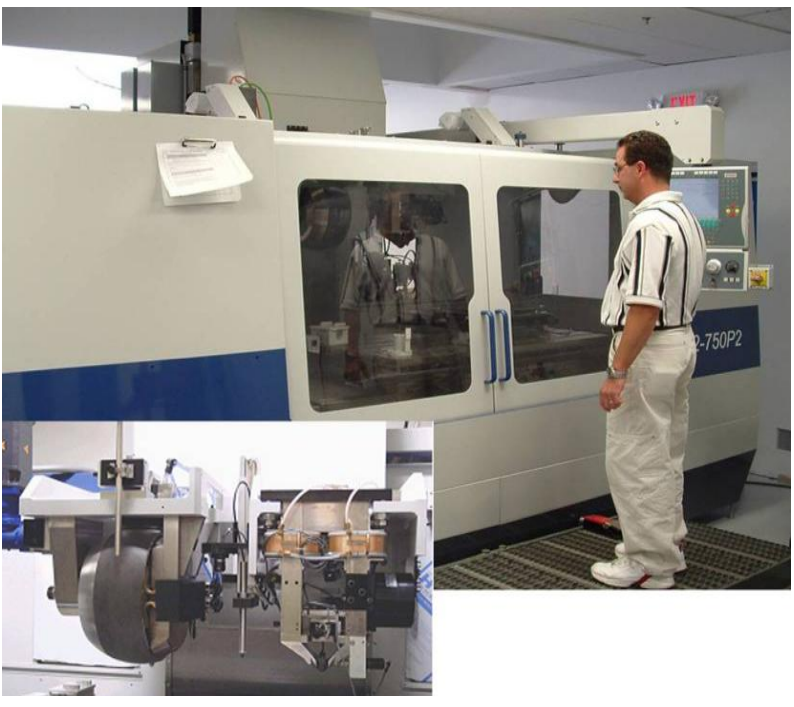

Figure 5: Large-aperture Q22-750P2 MRF system uses two wheels, one large and one small, to imprint topographical features down to spatial periods of $1 \mathrm{~mm}$. This machine can polish optics up to $750 \times 1000 \mathrm{~mm}$. 
rates $\left(\sim 1 \mathrm{~mm}^{3} / \mathrm{min}\right)$. Additionally, the process seamlessly fits into the advanced manufacturing process technology for production of large-aperture optical components possessing high-ultraviolet damage resistance. ${ }^{2,8}$ Today, we have 5 large-aperture MRF tools to perform large-aperture CPP imprinting and have fabricated over $350 \mathrm{CPP}$ imprints for NIF and other high-power laser systems.

\section{LASER RESISTANT LARGE-APERTURE OPTICS}

Laser-induced damage initiation on fused silica optics can limit the lifetime of the components when used in high-power UV laser environments. For example, in inertial confinement fusion research applications, the optics can be exposed to temporal UV laser pulses of about $3 \mathrm{~ns}$ with average fluences of $8 \mathrm{~J} / \mathrm{cm}^{2}$ and peak fluences between 12 and $15 \mathrm{~J} / \mathrm{cm}^{2}$. Laser-induced damage initiation and growth plays a key role in determining the operational lifetime and change-out rates for these optics. For large-aperture optics, optimization of UV damage performance is particularly important due to the surface area of the optic exposed to laser beam irradiation. The requirements necessary to minimize surface and subsurface damage on large optics used in megajoule-class laser systems, therefore, present unique manufacturing challenges that call for advanced finishing techniques and processes.

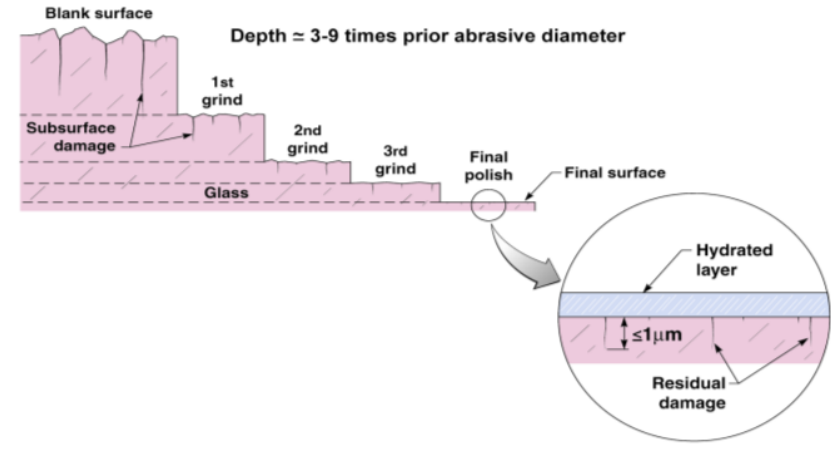

Figure 6: Schematic representation of an optical manufacturing process showing the changes in surface and subsurface damage from grinding through polishing process steps. The damage threshold of optics is determined by the tail of the fabrication defect population present on the completed optic. Material removal must be sufficient to eliminate subsurface damage originating from previous figuring and finishing operations.

zirconia-based polishing slurries ${ }^{13}$, conventional lap polishing inevitably results in both surface and subsurface mechanical damage due to the high normal loads present from the weight of the optic on the lap bed. Because of this, the subsurface damage layer persists regardless of how careful one is in controlling the process. Similar effects are observed with other types of conventional finishing such as small tool polishing.

In 2002, we introduced an advanced finishing process illustrated in Figure 7, that exhibited superior damage performance of fused silica at $351 \mathrm{~nm} .{ }^{8,14}$ The process uses MRF final finishing on high-purity "inclusion-free" fused silica substrates to attain final figure and superior damage performance once combined with $\mathrm{HF}$ acid etching to remove MRF
Damage initiation on fused silica optics in high fluence UV laser environments can arise from various manufacturing flaws and handling processes used during optic fabrication. Improper handling and cleanliness of the optics can cause damage initiation by introducing contaminants onto the optical surfaces. Such contaminants can absorb energy and initiate damage upon laser beam exposure. Similarly, the conventional polishing process itself can lead to the formation of damage sites. ${ }^{9,10,11,12}$ Such damage sites result from both surface and subsurface mechanical damage that is inherent to the high normal loads associated with conventional lap polishing as illustrated in Figure 6. In addition to the mechanical damage itself, such surface and subsurface cracks can serve as sites that can trap optically absorbing species, such as iron, ceria, and other contaminants, which are typically present in the polishing process. While improvement in damage initiation density can be realized by utilizing

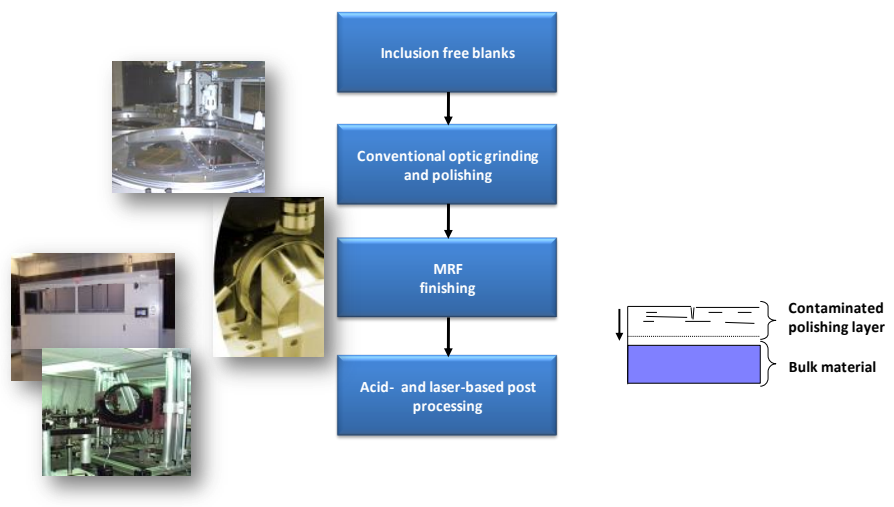

Figure 7: Recipe for MRF process to improve laser damage resistance. The general approach is to eliminate subsurface damage and near surface contamination using MRF and post-processing such that the surface and near surface "look" like bulk material. 
related contaminants such as iron and ceria. $\mathrm{MRF}^{15,16,17,18,19,20,21}$ is an advanced polishing technique that can finish optics without propagating the subsurface damage layer. The technique also removes preexisting subsurface damage under the correct conditions. The removal of subsurface damage using MRF can be attributed to the small normal stress applied to the glass surface compared to large shear stress which is created by the interaction of the tool's magnetic field and the MR fluid through the converging gap between the optic and tool's pole faces.

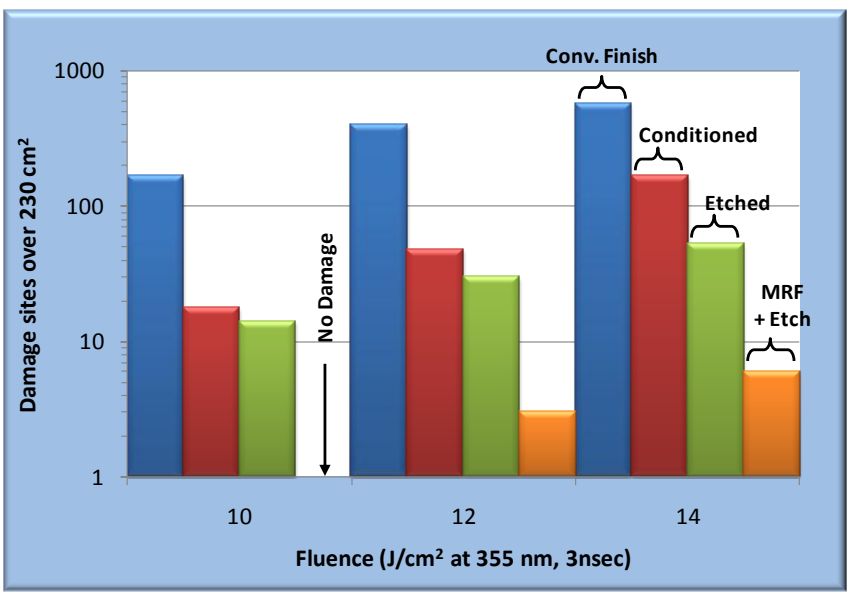

Figure 8: Comparison of damage test results for conventionally finished optics with and without MRF final polishing. MRF final polishing, when combined with appropriate post-processing, significantly reduces the damage sensitivity for high-fluence UV laser light.
Large-area damage tests conducted on optics finished with and without MRF final polishing show that MRF, when combined with appropriate post-processing, significantly reduces the damage sensitivity for highfluence UV laser light as illustrated in Figure 8. This is true regardless of the quality of the original conventional finishing. MRF can successfully remove subsurface damage and the contaminants contained therein leaving a high quality surface with little to no subsurface pits and cracks that could interact with the laser beam to induce damage. Even though MRF is successful at removing subsurface damage, the final MRF polish generates a new polishing layer containing MR fluid components at levels that are sufficient to promote damage. The MRF-induced polishing layer can be successfully removed via etching to yield superior performance. This is in contrast to the subsurface layer generated by conventional polishing which contains debris and a large number of pits and cracks which lead to a high probability for retention of optically absorbing species that can initiate damage when irradiated at high fluence.

From the cosmetic point-of-view, both edge-lit full aperture inspection and dark-field microscopy of the etched parts show that MRF finishing results in a dramatic reduction in subsurface damage when compared to that present on conventionally polished parts as illustrated in Figure 9. The large number of scatter sites and "chatter" on the
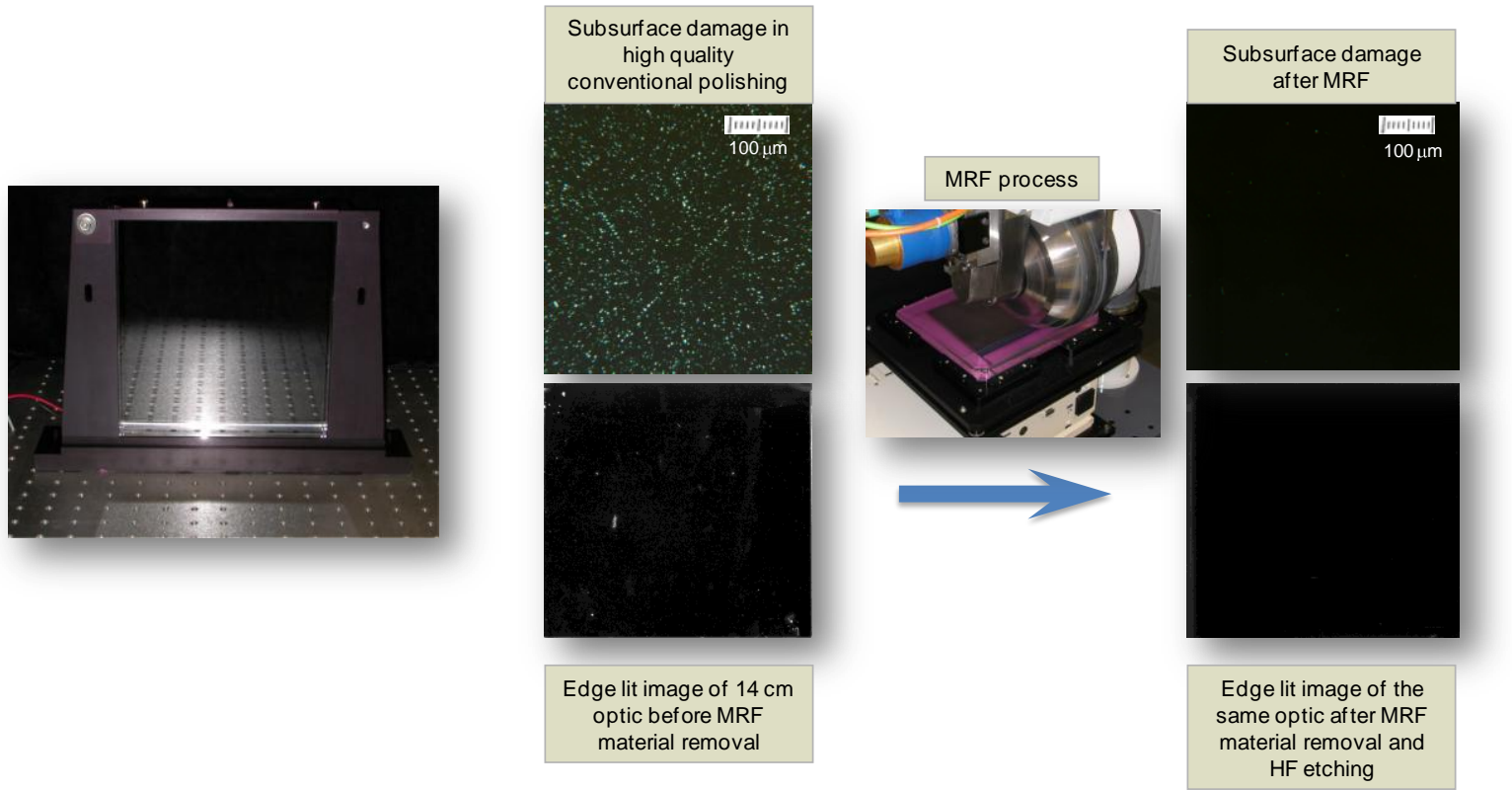

Figure 9: MRF polishing ${ }^{2,8,14}$ results in a dramatic reduction in subsurface damage compared to conventional polishing as shown in both dark-field microscopy and edge-lit damage maps before and after MRF polishing and etching. Subsequent laser damage testing to $14 \mathrm{~J} / \mathrm{cm}^{2}(351 \mathrm{~nm}, 3 \mathrm{~ns}$ equivalent) resulted in elimination of laser initiation sites. 
conventionally lapped optical surfaces indicates that the subsurface damage layer is significant in spite of care taken to manage the damage during polishing. It also shows that the large number of sites lead to a high probability for retention of absorbing species that can initiate damage when irradiated at high fluence. In contrast, the etched MRF polished optical surfaces show a near absence of subsurface damage that explains the large improvement observed in damage performance when compared to the best performing conventionally polished optics.

With the development and fielding of the large-aperture MRF tools, production process development and vendor technology transfer geared at manufacturing large-aperture damage resistant optics was approached to optimize the specific processes needed to ensure highest quality finished parts. The main goal here is to develop a manufacturing process for largeaperture thin (10-15 mm thick) fused silica optics capable of operating at 351-nm laser fluences up to $14 \mathrm{~J} / \mathrm{cm}^{2}$. This manufacturing process is designed around a detailed knowledge of subsurface damage arising from each process step in the manufacturing line. ${ }^{22,23,24,25}$ During the development phase of this effort, subsurface damage was measured for each of the process steps, including cleaning, handling, grinding, and polishing, using MRF wedge techniques. ${ }^{24}$ Different process machine parameters, coolants, abrasive types, and pads were analyzed, selected, and optimized to take advantage of high material removal rates with minimum or manageable subsurface damage. Once the subsurface damage characteristics were obtained for each step, minimum material removal requirements were put

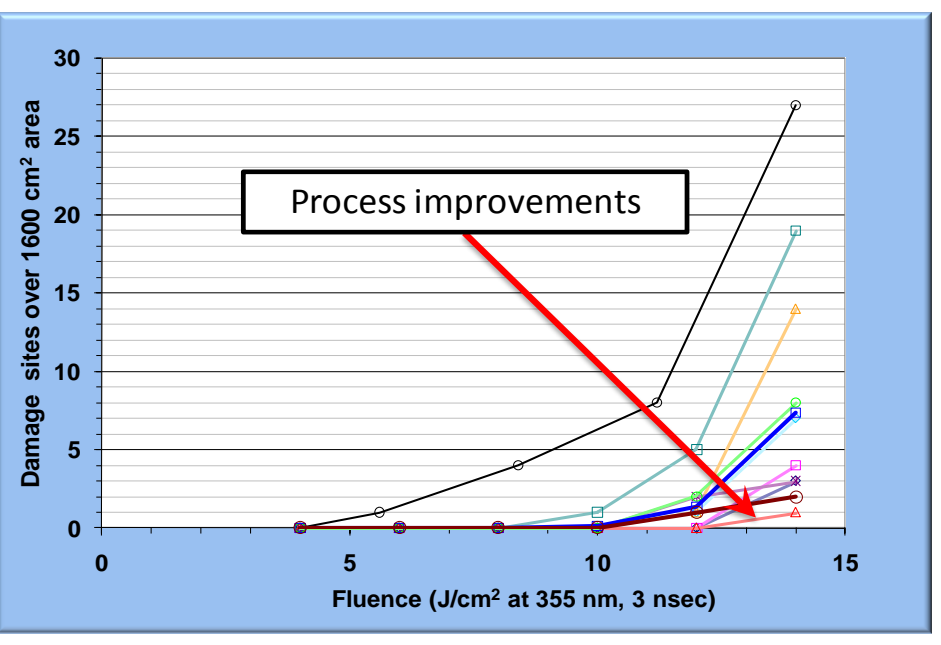

Figure 10: Laser damage test results for large-aperture thin fused silica optics and imprinted CPPs illustrate the success of optimizing optical fabrication processes and advanced MRF protocols. into place on subsequent steps to completely remove subsurface damage from previous steps. (refer to Figure 6). Additional material removal beyond the minimum implemented in each step enabled us to build in safety factors to ensure obtaining a finished optic with minimal or no subsurface damage. MRF final figuring and HF acid etching form part of this fabrication process. The damage test results of several large-aperture optics, main debris shields, CPP substrates, imprinted CCPs, and diffractive grating substrates, tested during this development effort are shown in Figure 10. Continued improvement in damage performance was observed over the development effort with the final process demonstration tests showing the greatest improvement at laser fluences of $12 \mathrm{~J} / \mathrm{cm}^{2}$ and greater. The goal of less than 50 growing sites per optic at $14 \mathrm{~J} / \mathrm{cm}^{2}$ was exceeded during production development and numerous demonstrations yielded optics having less than 8 sites. The improvement is directly attributed to optimizations made to the manufacturing process as a result of understanding and eliminating/managing subsurface damage at each step.

\section{MRF AS A TOOL TO MEASURE AND UNDERSTAND DAMAGE}

Management of fractures and subsurface mechanical damage (SSD) from processes used during optic fabrication plays a vital function in determining the final quality of an optic's surface finish. Brittle grinding processes induce fractures at, or near, an optical surface whose range can extend from depths of a few $\mu \mathrm{m}$ to hundreds of $\mu \mathrm{m}$. These process-induced or process-related fractures not only determine the current state of the optic in the fabrication process, they dictate how much material needs to be removed during subsequent $\operatorname{steps}^{22-25}$. The nature and extent of this damage also determines what manufacturing methods will need to be used to ultimately yield a completed optic having a superior surface finish. On the manufacturing floor, lack of knowledge or an insufficient understanding of process-induced fractures all too often leads to insufficient material removal during intermediate fabrication steps. This can result in occurrence of fractures and imperfections in the final optic, can cause the optic to have to be taken backwards in the manufacturing process for rework, or can add time to subsequent fabrication steps, particularly polishing, that reduces productivity and increases 
costs. From a functional perspective, fractures and SSD ultimately limit the performance of the optic under high stress conditions including high pressure, vacuum, large thermal gradients, and intense laser light. This is particularly important on optics containing flaws that have been hidden beneath a layer of re-deposited and modified material (usually weakly-structured hydrated material). In this situation, buried SSD can pose a serious problem because of its interaction with the optic's surroundings and sources of activation such as short-wavelength, high-intensity monochromatic light.

Significant advances have been made in developing diagnostic techniques that combine MRF and scanning optical microscopy to statistically measure and characterize SSD in optical materials. The method is called the MRF Wedge

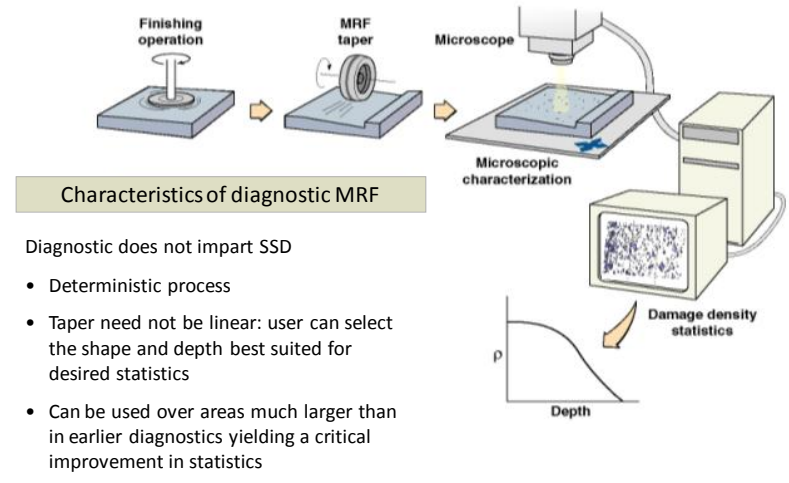

Figure 11: Schematic flow of MRF Wedge Technique used to measure fracture network distributions in optical surfaces. Prepared specimens are wedged polished using the MRF and then analyzed using an automated microscope. Technique. The technique is deterministic, making it easily controllable in removing a prescribed amount of material from an optical surface. Moreover, it is reproducible and can be applied repeatedly to a high degree of precision with depth and form control to better than $20 \mathrm{~nm} \cdot{ }^{22,23}$ MRF polishing has also been shown to expose and remove fractures without adding or propagating SSD. ${ }^{24}$ This makes it possible for one to evaluate a fabrication step without having to worry about collateral damage from sample preparation or measurement. The MRF Wedge Technique can be applied to large areas on the optic surface that enables one to obtain statistical information on the characteristics of fracture and flaw networks. The method is designed to be used on parts that have been through the same fabrication conditions as parts being produced in a manufacturing line. It also gives information regarding the profile of the fractures moving from the surface into the bulk in addition to the maximum depth of damage in an optic that is at a particular point in the fabrication process.

The basic concept behind the MRF Wedge Technique is to precisely polish a wedge into the surface of an optic to reveal the fracture network or imperfections present as a function of lateral distance along the wedge. The lateral distance is associated with the depth of the artifacts through knowledge of the wedge contour. This essentially provides information on the fracture distribution present as a function of depth by spreading the distribution laterally along the wedged surface. The MRF Wedge Technique consists of three steps shown in Figure 11. The first step involves obtaining optic specimens representing the manufacturing process needing to be characterized. These specimens can be obtained in a variety of ways. They can be drawn from a specific step of a manufacturing process, such as a particular grinding or polishing operation, as actual work pieces, by using surrogate samples processed in the same manner as production pieces, or they can be cut from actual production work pieces. As an example, process-related SSD data obtained using the MRF Wedge Technique shows quantitatively how SSD varies with abrasive size and type for different types of loose

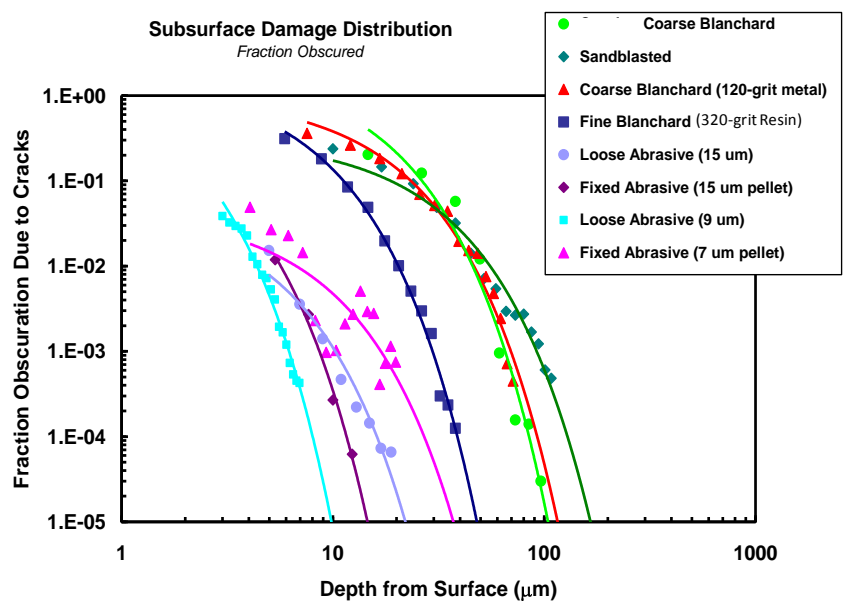

Figure 12: Measured fracture depth distributions for fused silica surfaces that have been treated by a wide variety of grinding processes. With this knowledge, fabrication procedures can be defined and optimized to manage SSD for each step required in an optical fabrication process. and fixed abrasive grinding processes as shown in Figure 12. The technique yields direct observation of the fracture depth and length distributions present in various common grinding processes and has been shown to be a valuable tool 
when used to study the details of fracture networks and imperfections in optical materials. The measurements conducted on the various grinding processes point out that the fracture networks are unique to each process and cannot be estimated with certainty given "general optical shop rules". The MRF Wedge Technique enables one to characterize each step in the fabrication process and to design and optimize process steps which may lead to more cost effective processes and higher quality optics.

Our experience in conventional finishing suggests that every process used to fabricate an optic imparts some level of damage at, or near, the surface. Detailed knowledge of the required material removal at each step is a necessary condition to avoiding damage propagation into subsequent process steps or damage "pile up" in a finished optic from processes early in the fabrication protocol. This knowledge needs to cover the entire fabrication process from blank to finished optic.

\section{NOVEL MATERIAL POLISHING PROCESSES}

Extremely hard, extremely soft, single-crystal, polycrystalline, or water-soluble optical materials play a significant role as critical optical components in lasers systems requiring high power. Use of these materials, however, presents unique challenges to the optical finishing processes required to meet stringent performance specifications. For example, a single-crystal material such as titanium doped sapphire (Ti:sapphire) has become the premier material for solid-state femtosecond high-peak power laser systems because of its wide bandwidth wavelength tuning range. With a tunable range from 680 to $1100 \mathrm{~nm}$, peaking at $800 \mathrm{~nm}$, Ti:sapphire lasing crystals can easily be tuned to the required pump

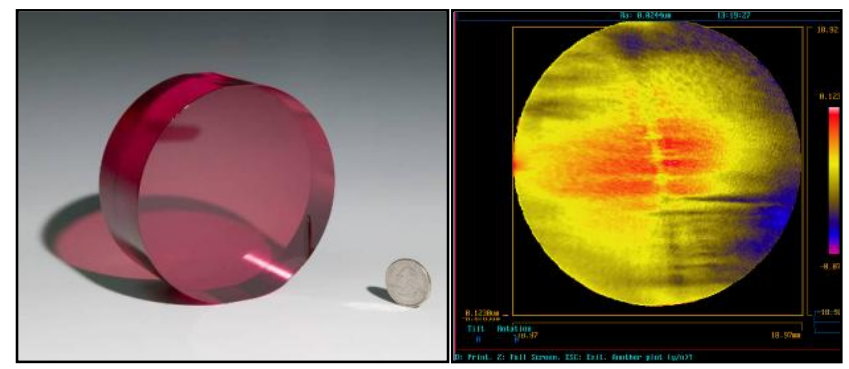

Figure 13: Left-105 mm diameter x $46 \mathrm{~mm}$ thick Ti:sapphire optic manufactured from a $150 \mathrm{~mm}$ crystal boule. Righttransmitted wavefront interferogram showing lattice distortion that can appear in sapphire and Ti:sapphire crystals. Peak-tovalley of $179 \mathrm{~nm}, \mathrm{rms} 29.9 \mathrm{~nm}$. wavelength and provide very high pump brightness due to their good beam quality and high output power of typically several watts. Extension to higher energies is limited by the size of the crystal lasing medium. Yields of high-quality large-diameter crystals have been constrained by lattice distortions and striations that may appear in the boule limiting the usable area from which high quality optics can be harvested. ${ }^{26}$ These imperfections manifest themselves as localized refractive index changes in the crystal's interior that deteriorate its transmitted wavefront quality even though the surfaces are made extremely flat. Distortions can vary from about $0.3-5 \mathrm{~mm}$ in width as shown in the transmitted wavefront in Figure 13. This distortion is large enough to disrupt the quality of a laser beam, which can cause damage to optics downstream in a laser system, and for short pulse systems can lead to incomplete compression and poor ability to focus the laser beam. As a result, any laser optic of Ti:sapphire that has these types of lattice distortions is less desirable for applications that require superior transmission characteristics and beam quality. To make matters even more complicated, Ti:sapphire is extremely hard (Mohs hardness of 9 with diamond being 10) which makes it extremely difficult to accurately polish using conventional methods without subsurface damage or significant wavefront error.

In the area of soft materials and water soluble materials, Ytterbium-doped strontium fluoroapatite (Yb:S-FAP) and potassium dihydrogen phosphate (KDP) single crystals are specialty optics that find use in high-power laser systems as laser light amplifying media (Yb:S$\mathrm{FAP})^{27,28}$ and frequency conversion and polarization switching media $(\mathrm{KDP})^{29}$. Both of these crystals are extremely difficult to grow in large sizes because of the introduction of contaminants and internal lattice imperfections that affect material performance and

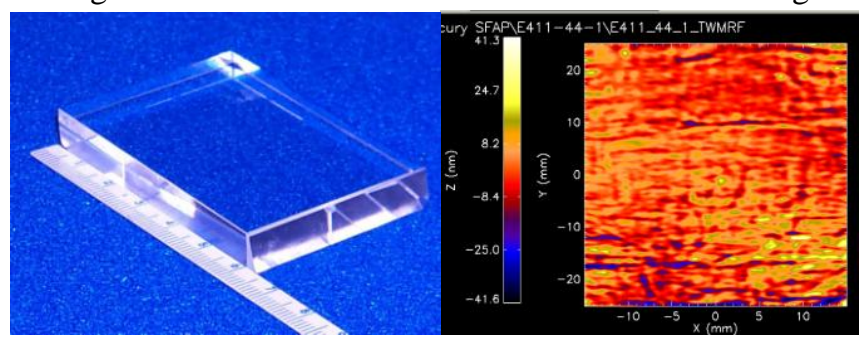

Figure 14: Yb:S-FAP crystals can contain grain boundaries that will disrupt the transmitted wavefront. Left: 40 X $60 \mathrm{~mm}$ crystal. Right: Transmitted wavefront interferogram of crystal showing mid-frequency grain boundaries. 
optical wavefront characteristics as shown in Figure 14. It is difficult to polish high-aspect ratio parts made from these materials with conventional pitch-lapping techniques. They are both very soft (Mohs hardness of 2.5) and flexible, temperature sensitive, prone to fracture, and scratch easily. KDP has further complication in that it is extremely water soluble (33g per $100 \mathrm{ml}$ ) thus requiring nonaqueous polishing media. Single-point diamond turning (SPDT) is considered state-of-the-art for KDP finishing. ${ }^{30}$ This process is capable of producing $44 \mathrm{~cm}$ flat plates for use in large laser systems. SPDT is done while showering mineral oil over the optic. This provides lubricity for cutting and temperature control. The oil is removed from the KDP surface with toluene or xylene.

Magnetorheological finishing (MRF) techniques have been developed as a proven method for compensating for slowlyvarying long-scale length $(10-50 \mathrm{~mm})$ lattice distortions and refractive index variations in glass and crystalline materials to provide for low transmitted wavefront distortion in plano-optics. ${ }^{31}$ In particular, MRF has made a significant

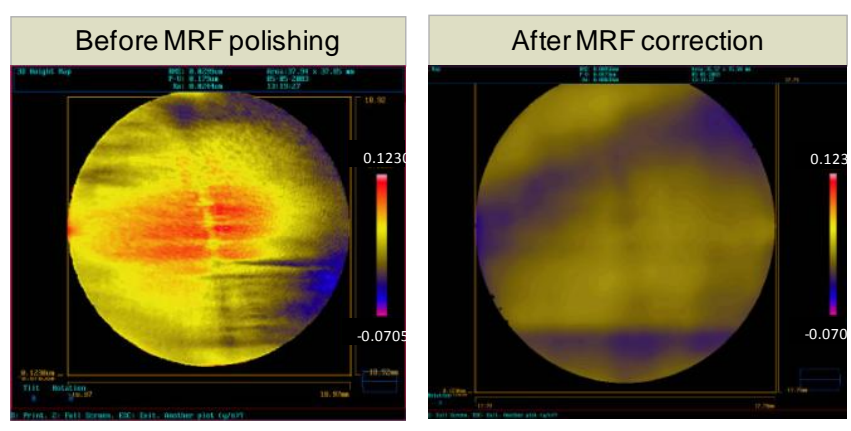

Figure 15: High quality Ti:sapphire. Left-transmitted wavefront of a lattice distortion that can appear in sapphire and Ti:sapphire crystals. Peak-to-valley of $179 \mathrm{~nm}$, rms $29.9 \mathrm{~nm}$. Righttransmitted wavefront after MRF shows a $3.8 \mathrm{x}$ improvement in Peak-to-valley $(47.3 \mathrm{~nm})$ and $3.7 \mathrm{x}$ improvement in rms $(9.0 \mathrm{~nm})$. contribution in compensating for low angle grain boundaries in $\mathrm{Yb}: \mathrm{S}-\mathrm{FAP} \quad[\mathrm{Yb} 3+: \mathrm{Sr} 5(\mathrm{PO} 4) 3 \mathrm{~F}]$ and Ti:sapphire crystals. Commercial MRF capabilities only compensate for long spatial period phase distortions of 3 $\mathrm{mm}$ or greater. We have developed MRF techniques to compensate for the sub-millimeter lattice distortions of sapphire and Ti:sapphire crystals to improve the transmitted wavefront. ${ }^{32}$ Our efforts in this area involve developing expertise and MRF equipment capabilities to correct for shorter period phase distortions and discrete inhomogeneities that can be applied in a unique manner to both glass and crystalline materials. MRF machine improvements involve novel topographical gradient fitting routines, polishing protocols, and specialized operating conditions that can deterministically correct discrete optical errors in the sub-millimeter range to improve the transmitted wavefront quality. Central to this development is the design and introduction of fiducialized MRF fixtures which accurately locate interferometric features at an absolute location in the optical plane, interferometric manipulation algorithms to relate fiducial locations to interferogram locations, enhanced fiducial camera system components that link fixtures and fiducials to within $3 \mu \mathrm{m}$ relative to MRF machine position, and the implementation of small and precisely controlled MRF removal functions. These improvements make it is possible to achieve low transmitted wavefronts in Ti:sapphire and sapphire crystals as illustrated by comparing the interferograms in Figure 15 where the process resulted in marked wavefront improvement of 3.8 times in peak-to-valley and 3.7 times in rms.

Another example showing how improvements in interferometric and MRF tool positional control can lead to enabling technological breakthroughs is shown in Figure 16. Here, advanced MRF techniques are used to correct for long-scale localized transmitted wavefront error in $\mathrm{Yb}$ :SFAP crystals used as laser amplifying media in the MERCURY laser system. During early system development, these amplifiers were fabricated by diffusion bonding two half-size crystals together to form a single amplifier of sufficient size. After bonding and conventional polishing, the amplifiers exhibited a low refractive index around the bond line which made them unusable as they could not be corrected by conventional polishing processes.
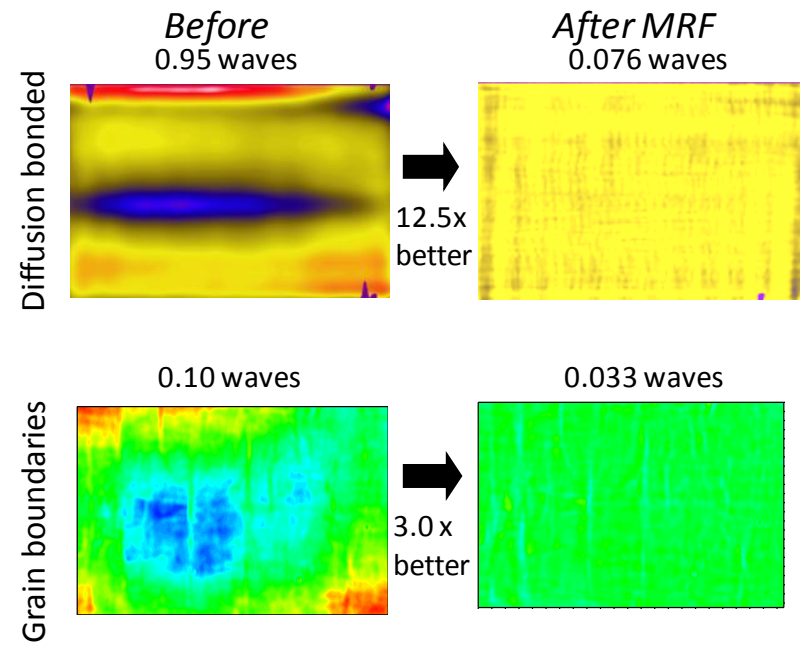

Figure 16: MRF imprinting technology applied to improve transmitted wavefront on a diffusion bonded Yb:S-FAP crystal amplifier. Note that long-scale bond line optical error has been eliminated revealing mid-frequency grain boundaries as the major optic error source. 
crystal's bulk, or interior. The MRF polishing protocol developed is used to correct for the low refractive index error in the crystal by MRF polishing the inverse of the optical artifact into one of the optical surfaces to yield an amplifier with wavefront properties sufficient to be used in the laser system. The outcome in this area has far reaching consequences: 1) the technology can be used to correct optics that would otherwise be unusable because they cannot be corrected using conventional processes, and, 2) the technology improvements can be applied for use in successfully fabricating optics from currently deficient materials where size restrictions or material quality impacts optic fabrication to the desired end.

Today, KDP crystals are finished using SPDT tools and nonaqueous lubricants/coolants. KDP optics fabricated using SPDT, however, are limited to surface corrections due to tool/method characteristics with surface quality driven by microroughness from machine pitch, speed, force, and diamond tool character. Though highly successful, SPDT can only be used to produce a flat surface on each of the KDP optical faces which results in having to select a crystal blank having bulk homogeneity properties adequate for production of an acceptable final optic. Moreover, one essentially has to live with the bulk crystal inhomogeneities that are present in the final optic and the resulting wavefront. The SPDT process is also only capable of yielding surfaces with 1.0- to 3.0-nm rms roughness which leads to an optical surface finish performance disadvantage when compared to other optic types where $0.2-0.5 \mathrm{~nm}$ rms roughness is typical. Mounting fixture and SPDT tool marks can also be problematic in these optics particularly when used in high laser fluence applications since they comprise a set of surfaces flaws that can damage and limits the optic's usable life. Advanced MRF technology can offer a new and novel means of finishing water-soluble crystals such as KDP with optical properties superior to what is available today. MRF polishing offers a means to circumvent many of these issues since it is deterministic which makes the technique practical for surface and transmitted wavefront correction, is low force, and is temperature independent. What is lacking is a usable nonaqueous MR fluid that is chemically and physically compatible with KDP which can be used for polishing and subsequently cleaned from the optical surface.

We have developed a nonaqueous MR fluid that shows promise for final finishing of large-aperture KDP. ${ }^{33,34}$ The components of the MR fluid include a nonaqueous carrier liquid, carbonyl iron spheres, a stabilizer, and a chemical buffer. Successful polishing of KDP crystal surfaces using MRF techniques requires a specifically tailored nonaqueous MR fluid compatible with both the magnetically active environment and the physical and chemical properties peculiar to KDP. Particular attention must be paid to several characteristics to make MRF polishing possible. These characteristics include: 1) carrier liquid type, 2) fluid stabilizer compatibility, 3) intentional or environmental water content, 4) carrier liquid content, and 5) MR fluid $\mathrm{pH}$. Compatible nonaqueous carrier liquids that form the basis of these fluids must be able to support dissolved KDP and/or water to keep the KDP removed from the crystal surface during polishing from redepositing back onto the crystal surface. Appropriate stabilizers must be used in the fluids to protect the carbonyl iron and the KDP crystal from reactions leading to fluid instability and particle agglomeration that produce poor surface finish from artifacts such as polishing-induced scratches and digs. The fluid must also be maintained between $\mathrm{pH}$ values

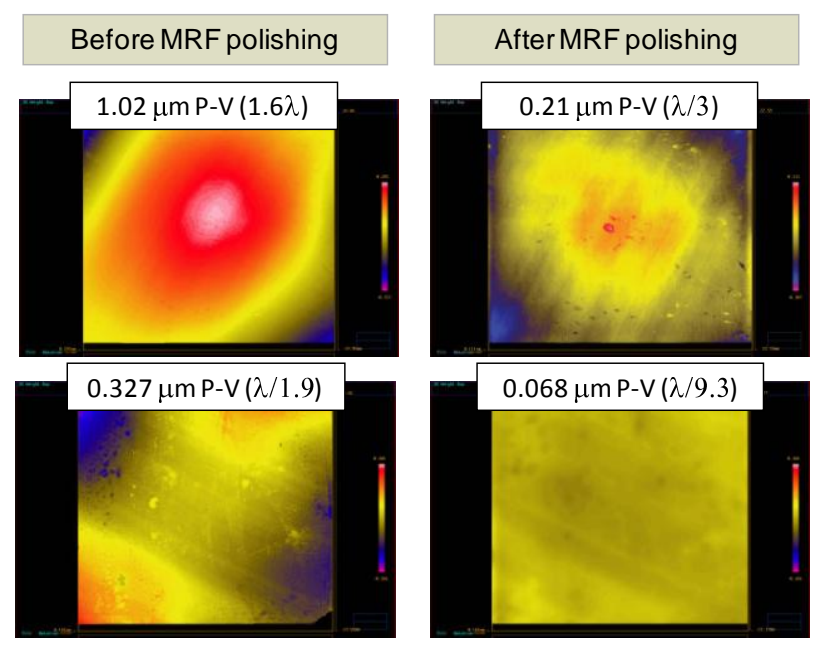

Figure 17: Surface interferograms of KDP samples before and after MRF polishing using nonaqueous MR fluids. Single pass MRF polishing improved surface figure by 5 times in both cases. of 6.5-9.0 to eliminate surface oxidation, particle agglomeration, and uncontrollable KDP dissolution via acidbase reaction where significant quantities of $\mathrm{PO}_{4}^{-3}$ are produced. Nonaqueous MR fluid performance can be optimized to produce mid-Angstrom level microroughness on KDP surfaces by adjusting the carrier liquid content.

Surface interferograms from MRF polishing tests on KDP specimens using the developed nonaqueous MR fluid are presented in Figure 17. The interferograms on the left show the incoming surface figure resulting from SPDT, one with a large peak-to-valley $(\mathrm{P}-\mathrm{V})$ of $1.02 \mu \mathrm{m}$ and the other with a small P-V of $0.327 \mu \mathrm{m}$. The small spots within the interferogram apertures are from non-uniform tape adhesion on the rear surfaces of the KDP crystals. Vinyl protective tape is used on the rear surface to eliminate reflection from this surface in the surface interferogram. After single pass MRF polishing of the KDP crystal surfaces, the surface figure is improved by about 5 times, to $0.21 \mathrm{~mm} \mathrm{P-V}$ and $0.68 \mathrm{~mm} \mathrm{P}-\mathrm{V}$. This result is typical of the surface figure improvements made on a per pass basis using deterministic 
MRF polishing. Overall, the success of these polishing tests combined with the improvements in surface roughness made $2.72 \mathrm{~nm} \mathrm{rms}$ (SPDT) reduced to $0.65 \mathrm{~nm} \mathrm{rms}$. (MRF) shows promise for using MRF as a technique to final finish KDP crystals with improved optical figure and finish.

\section{SUMMARY}

Over the last eight years, the evolution and success of MRF technological developments in machine and process applications has driven its success in large-aperture optic fabrication. Megajoule-class laser systems requiring highquality ultra-precision optics take advantage of this cutting-edge technology to meet the stringent requirements needed for reliable performance in the demanding high-power operational environment. The precision and deterministic nature of MRF polishing has proven to be the enabling factor in the manufacture of ultra-precise high-fidelity CPP fused silica optics needed for laser beam conditioning and quality in these large-aperture laser systems. The developments made in this area have also been applied to correct optic error in other optical materials, such as crystals, that would otherwise make the optics unusable because they cannot be corrected using conventional processes. MRF's unique material removal mechanism also makes it possible to expose and remove surface and subsurface flaws in optics during final polishing to yield optics with superior UV laser damage resistance. This feature plays a key role in determining the operational lifetime and change-out rates for large-aperture optics due to the surface area exposed to laser beam irradiation and the need to have optics free of manufacturing and handling surface artifacts. MRF has also found a place as a tool for use in assessing and developing optic manufacturing processes by enabling one to measure and characterize damage present and attributable to specific manufacturing steps. Every process used to fabricate an optic imparts some level of damage at, or near, the surface. Detailed knowledge of the required material removal at each step is a necessary condition to avoiding damage propagation into subsequent process steps or damage "pile up" in a finished optic from processes early in the fabrication protocol. This knowledge needs to cover the entire fabrication process from blank to finished optic. Overall, MRF has proven to be a viable finishing process technology to enable and optimize performance in high-power laser systems and optical devices, and it will continue to evolve as more optical fabrication challenges and requirements are encountered in state-of-the-art applications.

\section{REFERENCES}

${ }^{1}$ J. A. Menapace, S. N. Dixit, F. Y. Génin, W. F. Brocious, Magnetorheological Finishing for Imprinting Continuous Phase Plate Structure onto Optical Surfaces, Laser Induced Damage in Optical Materials: SPIE Proc., 5273, 220-230 (2003).

${ }^{2}$ J. A. Menapace, P. J. Davis, W. A. Steele, M. R. Hachkowski, A. Nelson, K. Xin, MRF applications: on the road to making large-aperture ultraviolet laser resistant continuous phase plates for high-power lasers, Laser Induced Damage in Optical Materials: SPIE Proc., 6403, 64030N (2006).

3 J. A. Menapace, P. J. Davis, S. Dixit, J. H. Campbell, D. Golini, M. R. Hachkowski, A. Nelson, Imprinting continuously varying topographical structure onto large-aperture optical surfaces using magnetorheological finishing, Proc. euspen, II, 181-184 (2007).

${ }^{4}$ J. Paisner and J. R. Murray. Overview of the National Ignition Facility Project. Proc. SPIE Conf. Solid-State Laser Apps. for ICF (Monterey, CA 1998).

${ }^{5}$ P. Wegner, J. Auerbach, T. Biediada, S. Dixit, J. Lawson, J. Menapace, T. Parham, D. Swift, P. Whitman, and W. Williams. NIF final optics system: frequency conversion and beam conditioning. Proc. SPIE, 5341, pp. 180-189 (2004).

${ }^{6}$ S. N. Dixit, M. D. Feit, M. D. Perry, and H. T. Powell. Designing fully continuous phase screens for tailoring focalplane irradiance profiles. Optics Letters, vol. 21, no. 21, 1715-1717 (1 Nov. 1996). 
${ }^{7}$ Distributed phase plates for super-Gaussian focal plane irradiance profiles. LLE (Laboratory for Laser Energetics) Rev. 63, pp. 126-129 (1995).

${ }^{8}$ Menapace, J. A., Penetrante, B., Golini, D., Slomba, A., Miller, P. E., Parham, T., Nichols, M., Peterson, J., 2002, Combined advanced finishing and UV-laser conditioning for producing UV-damage-resistant fused-silica optics, Laser Induced Damage in Optical Materials: SPIE Proc., 4679, 56-68 (2002).

${ }^{9}$ Z. Gu, P. Liang, and W. Zhang, Influence of glass surface layers on laser-induced damage threshold, Laser Induced Damage in Optical Materials: SPIE Proc., 4679, 75-78 (2002).

${ }^{10}$ P. Bouchut, P. Garrec, J. Newport, P. Gacoin, F. Bonnemason, S. Kaladgew, Technological processing of silica as seen through laser induced damage tests, Laser Induced Damage in Optical Materials: SPIE Proc., 4679, 242-250 (2002).

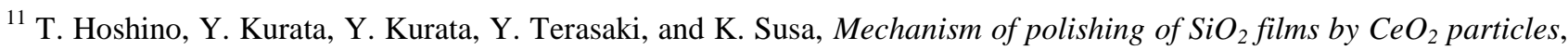
Journal of Non-Crystalline Solids, 283, 129-136 (2001).

12 M. J. Fluss, J. Plitzko, M. Wall, Initiation identification in 355-nm optics, Laser Induced Damage in Optical Materials: SPIE Proc., 4679, 17-22 (2002).

${ }^{13}$ D. W. Camp, M. R. Kozlowski, L. M. Sheehan, M. Nichols, M. Dovik, R. Raether, I. Thomas, Subsurface damage and polishing compound affect at the 355-nm laser damage threshold of fused silica surfaces, Laser Induced Damage in Optical Materials: SPIE Proc., 3244, 356-364 (1997).

14 J. A. Menapace, et al. Combined advanced finishing and UV laser conditioning process for producing damage resistant optics, U.S. Patent \# 6,920,765, July 26, 2005.

${ }^{15}$ D. Golini, S. Jacobs, W. Kordonski, and P. Dumas, Precision optics fabrication using magnetorheological finishing, Advanced Materials for Optics and Precision Structures, CR67, 251-274 (1997).

${ }^{16}$ A. B. Shorey, S. D. Jacobs, W. I. Kordonski, and R. F. Gans, Experiments and observations regarding mechanisms of glass removal in magnetorheological finishing, Applied Optics, 40, No. 1, 20-33 (2001).

${ }^{17}$ W. Kordonski, D. Golini, P. Dumas, and S. Jacobs, Magnetorheological suspension-based finishing technology, SPIE, 3326, 527-535 (1998).

${ }^{18}$ S. D. Jacobs, D. Golini, Y. Hsu, B. E. Puchebner, D. Strafford, W. I. Kordonski, I. V. Prokhorov, E. Fess, D. Pietrowski, and V. W. Kordonski, Magnetorheological finishing: a deterministic process for optics manufacturing, SPIE, 2576, 372-382 (1995).

${ }^{19}$ S. D. Jacobs. F. Yang, E. M. Fess, J. B. Feingold, B. E. Gillman, W. I. Kordonski, H. Edwards, and D. Golini, Magnetorheological finishing of IR materials, SPIE, 3134, 258-269 (1997).

${ }^{20}$ S. R. Arrasmith, I. A. Kozhinova, L. L. Greg, A. B. Shorey, H. J. Romanofsky, S. D. Jacobs, D. Golini, W. I. Kordonski, S. Hogan, and P. Dumas, Details of the polishing spot in magnetorheological finishing, SPIE, 3782, 92100 (1999).

${ }^{21}$ D. Golini, W. I. Kordonski, P. Dumas, and S. Hogan, Magnetorheological finishing (MRF) in commercial precision optics manufacturing, SPIE, 3782, 80-91 (1999).

22 P. E. Miller, T. I. Suratwala, J. P. Menapace, L. L. Wong, P. J. Davis, and W. A. Steele, The Distribution of Subsurface Damage in Fused Silica, Laser Induced Damage in Optical Materials: SPIE Proc., 5991, 599101 1-25 (2005). 
${ }^{23}$ T. Suratwala, L. Wong, P. Miller, M. D. Feit, J. Menapace, R. Steele, P. Davis, D. Walmer, Sub-surface mechanical damage distributions during grinding of fused silica, Journal of Non-Crystalline Solids 352 (2006) 5601.

${ }^{24}$ J. A. Menapace, P. J. Davis, W. A. Steele, L. L. Wong, T. I. Suratwala, and P. E. Miller, MRF Applications: Measurement of Process-dependent Subsurface Damage in Optical Materials using the MRF Wedge Technique, Laser Induced Damage in Optical Materials: SPIE Proc., 5991, 599103 1-9 (2005).

25 J. A. Menapace, P. J. Davis, W. A. Steele, L. L. Wong, T. I. Suratwala, and P. E. Miller, Utilization of Magnetorheological Finishing as a Diagnostic Tool for Investigating the Three-Dimensional Structure of Fractures in Fused Silica, Laser Induced Damage in Optical Materials: SPIE Proc., 5991, 599102 1-13 (2005).

${ }^{26}$ W. R. Rapoport, C. P. Kattak, Titanium sapphire laser characteristics, Appl. Opt. 27(13) 2677-2684 (1988). Also, a direct quote from the Crystal Systems website on Ti:sapphire.

${ }^{27}$ Zhi M. Liao; John J. Adams; Jeff Jarboe; Joe Menapace; Norm Nielsen; Sam Schrauth; Justin Wolfe; Al Erlandson; Kathleen Schaffers; Christopher Stolz; Andy Bayramian; John Caird, Laser-induced bulk damage in Yb:S-FAP crystals, Laser Induced Damage in Optical Materials: SPIE Proc., 6403, 64031X 1-6 (2007).

${ }^{28}$ Castaing; François Balembois; Patrick Georges; Thierry Georges; Kathleen Schaffers; John Tassano , Intracavity pumped Yb:SFAP crystal emitting at $985 \mathrm{~nm}$ and second harmonic generation, Proceedings of Solid State Lasers XVII: Technology and Devices, 5 March 2008.

29 Martynas Barkauskas; Andrius Melninkaitis; Darius Mikšys; Loreta Meslinaite; Rimantas Grigonis; Valdas Sirutkaitis; Hervé Bercegol; Laurent Lamaignère, Characterization of KDP crystals used in large aperture doublers and triplers, Laser Induced Damage in Optical Materials: SPIE Proc., 6403, 64031V 1-10 (2007).

${ }^{30}$ R. C. Montesanti and S. L. Thompson, Lawrence Livermore National Laboratory, Livermore, CA, UCRL-ID-121651 (1995).

${ }^{31}$ K. I. Schaffers, J. A. Menapace, et.al., Growth of large laser crystals for high power lasers, Third International Workshop on Crystal Growth Technology, Beatenberg, Switzerland, (Sept. 2005).

${ }^{32}$ J. A. Menapace, et. al., U.S. Patent Application: METHOD AND SYSTEM FOR PROCESSING OPTICAL ELEMENTS USING MAGNETORHEOLOGICAL FINISHING, Attorney Docket No.: 027512-002110US

33 J. A. Menapace, P. R. Ehrmann, R. C. Bickel, Magnetorheological finishing (MRF) of potassium dihydrogen phosphate (KDP) crystals: nonaqueous fluids development, optical finish, and laser damage performance at $1064 \mathrm{~nm}$ and $532 \mathrm{~nm}$, Laser Induced Damage in Optical Materials: SPIE Proc., 7504, 750414 1-12 (2009).

${ }^{34}$ J. A. Menapace, et. al., U.S. Patent Application: METHOD AND SYSTEM FOR POLISHING MATERIALS USING A NONAQUEOUS MAGNETORHEOLOGICAL FLUID, Attorney Docket No.: 027512-004310US 